\title{
Planning of HIFU therapies of moving organs by using numerical simulation techniques
}

\author{
Joachim Georgii", Caroline von Dresky, Daniel Demedts, Christian Schumann, Tobias Preusser \\ From 2nd European Symposium on Focused Ultrasound Therapy \\ Rome, Italy. 10-11 October 2013
}

\begin{abstract}
Background
We present a software prototype that allows for preinterventional planning and simulation of focused ultrasound therapies. By using numerical simulation techniques the heating and destruction of the tissue can be predicted and thus a patient-specific treatment plan can be generated in advance. Since our simulation is amenable to respect rib shielding, organ motion as well as focal point tracing, it could in particular support the planning of liver treatments.
\end{abstract}

\section{Material and methods}

The simulation of the ultrasound propagation is based on the hybrid angular spectrum method. The heating of the tissue is computed by solving the Pennes bioheat equation. Both simulations have been massively parallelized on graphics processing units to improve computation times. The simulation of the pressure field includes single reflection of the ultrasound beams at acoustically dense structures as the ribs. Technically, these reflections are computed by a so-called back-sweep of the spectral propagator. Moreover, motion of the tissue is incorporated in the simulation by allowing the material parameters to be updated in each simulation step. Additionally, heat transportation of moving tissue is respected in the bioheat equation solver. By adjusting the transducer parameters for electronic steering, we can simulate focal point tracing in moving organs. Based on the temperature field, we also predict the thermal damage by computing the Arrhenius damage model.

\section{Results}

Our approach allows for the simulation of entire treatment plans composed of a set of sonications and cooling phases. For a 40 s sonication simulated at a resolution of $256 \times 256 \times 256$ pressure samples and $128 \times 128 \times 128$

Fraunhofer MEVIS, Bremen, Germany 